\title{
Die Paul-Martini-Stiftung
}

Die Paul-Martini-Stiftung (PMS) wurde am 4. August 1966 von der Medizinisch Pharmazeutischen Studiengesellschaft (MPS) mit Sitz in Frankfurt a.M. gegründet. Diese Studiengesellschaft hatten einige Jahre zuvor einige führende deutsche Pharmafirmen als eine Art Think Tank gegründet (Bayer, Boehringer Ingelheim, Boehringer Mannheim, Hoechst, Knoll, Merck und Schering). Die MPS fungierte als Träger der Stiftung, bis 1994 der Verband Forschender Arzneimittelhersteller, der vfa, übernahm.

Die MPS benannte die Stiftung nach dem 1964 verstorbenen Bonner Wissenschaftler und Arzt Prof. Dr. med. Dr. med. h.c. Paul Martini. Dieser hat sich besondere Verdienste um die Methodik der klinisch-therapeutischen Forschung erworben und diese mit seiner 1932 veröffentlichten „Methodenlehre der therapeutischen Untersuchung“ über Jahrzehnte wesentlich geprägt. In dieser Methodenlehre hat er als erster randomisierte, kontrollierte klinische Studien für die Ermittlung der Wirksamkeit, der Sicherheit und der Risiken eines neuen Arzneimittels gefordert.

Zweck der Stiftung war und ist bis heute die Förderung der Arzneimittelforschung. In den ersten Jahren konzentrierte sich die Stiftung dabei auf die Fortbildung jüngerer Wissenschaftler vor allem auf dem Gebiet der klinischen Pharmakologie, auf dem Deutschland Nachholbedarf hatte. Für diesen Fokus gab es zwei wichtige Gründe: Zum einen ist die Arzneimittelentwicklung darauf angewiesen, dass es entsprechende Expertise an Hochschulen, Krankenhäusern und bei den Behörden gibt. Zum anderen ist diese Expertise die Voraussetzung für eine rationale und damit gezielte und effektive Arzneimittelanwendung. Von Anfang an kam in der Stiftung der Position des wissenschaftlichen Beraters eine bedeutende Rolle zu. Hierfür konnte die Stiftung stets anerkannte Mediziner gewinnen, die dank ihrer Weitsicht wegweisend die Stiftungsaktivitäten lenkten.

Unter ihrem ersten wissenschaftlichen Berater, Prof. Dr. phil. Dr. med. Dr. med. h.c. Wolfgang Wirth, konzentrierte sich die PMS auf die Vergabe von einjährigen Stipendien für klinische Pharmakologie, um entsprechende Expertise auch in Deutschland aufzubauen. Bis Anfang 1974 wurden insgesamt 28 Stipendien vergeben, wobei die Wissenschaftler ihre Fortbildung meist in den USA absolvierten. Es folgte die Etablierung der klinischen Pharmakologie an Universitäten, und die PMS förderte für 3 Jahre mit bis zu 100000 DM jährlich die Einrichtung klinisch-pharmakologischer Einheiten an den Universitäten Heidelberg, Mainz, Würzburg und Düsseldorf und Tübingen.
Unter Prof. Dr. med. Dr. med. h.c. Hans Erhard Bock wurden diese Aktivitäten fortgesetzt: Die PMS finanzierte von 1974 bis 1978 ein Schwerpunktprogramm zur Förderung der klinischen Pharmakologie an Hochschulen gemeinsam mit der Deutschen Forschungsgemeinschaft (DFG). 1979 empfahl die Gesundheitsministerkonferenz der Kultusministerkonferenz die Errichtung von Lehrstühlen und Schaffung von Abteilungen für Klinische Pharmakologie an den Universitäten, und der Deutsche Ärztetag verabschiedete das Teilgebiet Klinische Pharmakologie. Insgesamt wurden von der PMS 44 Vollstipendien und 36 Kurzstipendien vergeben.

Seit 1969 wurde die klinische Pharmakologie auch durch die Vergabe des Paul-Martini-Preises gefördert. Dieser war anfangs mit $5000 \mathrm{DM}$, ab 1971 mit 10000 DM dotiert und wurde zunächst gemeinsam mit der damaligen Deutschen Gesellschaft für Dokumentation und Statistik vergeben. Seit Einführung des Euro Anfang 2002 beträgt das Preisgeld 25000 Euro. Die PMS will mit dem Preis dazu beitragen, dass die oft im Schatten der Laborforschung stehende klinische Forschung öffentliche Aufmerksamkeit erhält. Die Verleihung des Paul-Martini-Preises findet im Rahmen des jährlichen Kongresses der Deutschen Gesellschaft für Innere Medizin (DGIM) statt.

Unter Federführung von Prof. Dr. med. Hellmuth Kleinsorge und Prof. Dr. med. Hans Josef Dengler entwickelte sich die Paul-Martini-Stiftung immer mehr zu einem Veranstalter wissenschaftlicher Begegnungen (Symposien, Workshops, Expertengespräche) von forschender Industrie, Universität, Klinik und Praxis. Genannt seien hier die in der Akademie der Wissenschaften und der Literatur in Mainz durchgeführten Symposien oder die Beiträge der Paul-Martini-Stiftung im Hauptprogramm der Jahrestagung der Deutschen Gesellschaft für Innere Medizin. Eigene Veröffentlichungen der Paul-Martini-Stiftung wurden ein fester Bestandteil der Stiftungsaktivitäten.

Anfang der 1990er Jahre wurden die Ziele der PMS angepasst: Die Arzneimitteltherapie und die klinische Pharmakologie stellten weiterhin einen Arbeitsschwerpunkt der Stiftung dar, aber andere Themen wie Kosten-Nutzen-Analyse und Lebensqualität sowie Pharmakoepidemiologie wurden als wichtig für die Therapieforschung identifiziert und in die Aktivitäten aufgenommen.

1994 nahm die PMS eine wichtige Hürde - den Wechsel der Trägerschaft von der MPS zum Verband Forschender Arzneimittelhersteller (vfa), zu dem die MPS ausgebaut wurde. 
Unter der Ägide von Prof. Dr. med. Dr. h. c. Peter C. Scriba erfolgte im Jahr 2000 der Umzug der PMS - im Gefolge des vfa - nach Berlin. 2004 gab es zwei bedeutende Änderungen: Seitdem werden die Herbstsymposien nicht mehr in Mainz in der Akademie der Wissenschaften, sondern in Berlin, meist in den Räumen der Kaiserin-Friedrich-Stiftung, durchgeführt und zwar in Verbindung mit der 1652 gegründete Academia Naturae Curiosorum, besser bekannt als Leopoldina; diese nimmt nach einem Beschluss der Gemeinsamen Wissenschaftskonferenz von Bund und Ländern seit 2008 die Aufgaben der deutschen Nationalen Akademie der Wissenschaften wahr.

Seit 2012 wird die PMS von Prof. Dr. med. Stefan Endres wissenschaftlich beraten, der mit der Einführung von Reisestipendien für junge Wissenschaftler und der vermehrten Ausrichtung von Workshops zu hoch aktuellen Forschungsthemen wie Oligonukleotiden, Immunonkologika und Gen-basierten Therapien eigene Akzente gesetzt hat.

Die PMS wird auch weiterhin gemäß ihrem Stiftungszweck den wissenschaftlichen Dialog zwischen medizinischen Wissenschaftlern in Universitäten, Krankenhäusern, den forschenden Pharmafirmen, anderen Forschungseinrichtungen und Vertretern von Gesundheitspolitik und Behörden intensivieren. Damit will sie auch dazu beitragen, den Forschungsstandort Deutschland zu stärken. 